\title{
A Study on Knowledge Quality and Job Performance of Knowledge Workers by Analyzing Content of Social Network Sites Using Sentiment Network Analysis
}

\author{
Renn Aun Justin Lim, *Siew Hoong Angela Lee, Tong-Ming Lim \\ Sunway University, Malaysia \\ *angelal@sunway.edu.my
}

\begin{abstract}
The importance of knowledge quality has become a key research area in recent research works. Many organizations realize that benefits of knowledge creation and sharing cannot be obtained unless quality of knowledge is achieved. This research investigates the perception of knowledge workers that uses social network sites as a means for social interaction, collaboration and knowledge sharing. The entries posted by these knowledge workers on the social network sites are gathered and studied with the presence of the element of sentiment such as emotion using sentiment network analysis. Based on the analysis, the results investigate the relationship between sentiment and quality of knowledge shared among knowledge workers on social networking sites and individual job performance. This research will analyse the improvements of employees' job performance through the quality knowledge using interview, questionnaire survey, and text analysis to look for conversational patterns and element of sentiment. This research will investigate a number of knowledge driven companies that use social network sites. Structural Equation Modelling (SEM) is used to analyse responses collected from survey data.
\end{abstract}

Keywords: Knowledge Sharing, Knowledge Quality, Job Performance, Social Network Sites, Sentiment Network Analysis

\section{Introduction}

Web 2.0 has taken the world by storm with its collaborative and dynamic network characteristics. Paul Grabowicz (2013) described Web 2.0 as the transition of static web to user oriented web where the culture of public participation as the key to the power of collective intelligence. By moving away from the static and passive audience, Web 2.0 technologies promote a collaborative culture on a social network site encouraging active communication or participation with other participants regardless of distance and location. It is accurate to say that Web 2.0 technologies bring the emergence of user oriented social media sites that couple video or picture in addition to plain text messages. These socially shared sites include blogs, wikis and social networking sites like the popular Facebook. For those companies that adopted Web 2.0 technologies into their workflow process find that social network sites are useful as knowledge sharing platforms. Some Web 2.0 deployments such as Yammer or Work Voices are designed specifically for enterprises' use. These enterprise micro blogging sites provide as platforms for employees to share knowledge within the company. These sites are appropriate for internal use because it ensures confidentiality where employees are able to share sensitive information with each other pertaining to the company's operation.

Liu (2010) stated that opinions are sentiments which can be subjective manifestation of people's feeling towards a specific event in textual information. Therefore, analysing the opinions or sentiments of content on the social network sites can provide a lot of information to the company. In the works conducted by Prabowo and Thelwall (2003), they found that sentiments are significant indicators for many different purposes which can be found in comments. Thus, these social network sites provide as channels for unbiased opinion to be expressed publicly and freely. This research studies the use of social network sites adopted by companies for the purpose of knowledge sharing. The content on the social network sites will be investigated. The element of sentiment between in the comments posted by participants will be investigated. The influence of sentiment embedded in the posts to the knowledge sharing attitude among knowledge workers and impact to their individual performance is examined. In this research, interview and questionnaire survey will be conducted and data that will be gathered and analysed. Sentiment network analysis will be used to study the posts compiled on the social networking sites. Text analytics will be used to study relationships between participants and sentiment analysis will be used to examine its influence toward the quality of knowledge and how such quality affects their individual performance. 


\section{Literature Review}

This research studies the relationship between individual job performance and the quality of knowledge posted by knowledge workers on Web 2.0 systems using sentiment network analysis. For knowledge shared by knowledge workers to bring some values to the employees and the company, posts must contain knowledge of some quality and align to the company's goals. Understanding the motivational factors that encourage the employees to contribute and share knowledge is essential. A good understanding on the Web 2.0 as knowledge sharing tools that are used to communicate and collaborate with peers with organizations is another critical area that needs to be researched on. The features offered by Web 2.0 support close social interaction through highly intuitive and easy to use interfaces. These characteristics have offered knowledge workers another platform to share knowledge. In a review conducted by McKinsey Global where the survey data was analysed by Jacques, Chui and Miller (2009), they found that companies have benefited from Web 2.0 technologies usage. With Web 2.0 technologies, there has been an increase in the number of users and companies that have started using Web 2.0 for business purposes. In short, companies which are using Web 2.0 technologies regardless of any industry would likely to report measurable benefits from using Web 2.0 technologies as knowledge management platform.

In another piece of work conducted by Gunther et al (2009), these authors model micro blogging adoption in enterprises to understand its influence in organizations. Micro blogging is an implementation of Web 2.0. Examples of micro blogging are Twitter and Yammer. Through the use of Twitter and Yammer, the authors conducted four focus groups to determine users' acceptance and usage of micro blogging system in their workplaces. According to the authors, adopting micro blogging concepts into the organization is beneficial since the usage of Twitter has proven to be a successful medium of communication in the investigation. This shows that the amount of communication and collaboration among users on micro blogging sites such as Twitter and Yammer has increased. Companies are able to derive limitless amount of new knowledge from these sites on knowledge workers' communication and knowledge shared with each other. Moreover, based on the study by Grit (2009), the author also used micro blogging in his research. He conducted a pilot test for different companies using different social network platforms. One of them uses Work Voices whereas the other uses Yammer. The authors use questionnaire to gather data from a number of employees within companies and based on the result of the study, it shows that the use of micro blogging actually indicates that there is an increase access to knowledge and also the intention to share knowledge.

In the work conducted by Costa et al (2012), the authors conducted a research based on the integration of online social networks and business intelligence. The authors stated that there are two main areas of research that analyses the information on social networking sites: Social Network Analysis which consists of Link Analysis and Web Mining and Sentiment Analysis which provides analysis on text or unstructured mining. Sentiment Network Analysis looks at Link analysis, web mining and unstructured text analysis. The later would prove to be a more comprehensive research as social networking sites contains comments or posts published by the users in text or unstructured languages. Therefore using sentiment analytical approaches such as text mining would provide a much more detail analysis. The authors conducted a case study to test out their architecture which involves grabbing data from Twitter based on a specific topic. Once obtaining the data, the filtering of the tweets are matched against the topic. The information goes through data cleansing process. The tweets are narrowed down to 58,906 tweets from 26122 users. The authors use numerous scoring systems to indicate the impact of the tweets and the influence of the tweets on the topic. Therefore, the unstructured data are organised and stored in the data warehouse as structured data. After creating the data warehouse and business intelligence integration, the authors uses QlikView software to produce reports for comparison. The reports show the influence of the tweets versus organizational factors such as total sales, location and others. Based on the author's research outcomes, the analysis on social network through the unstructured data mining approaches is proven to be beneficial towards the departments in the organization. It can better facilitate the planning and marketing of these departments. This shows that social networking analysis provides the means of unlimited information or knowledge gathering which are very useful for organizations. Furthermore, by mining the data and converting it into meaningful information or knowledge, it produces results which can help the organization in many ways such as improving sales, enhancing product, gathering positive and negative feedbacks. In conclusion, social networking sites are suitable platforms for knowledge sharing and by conducting sentiment network analysis on these sites; they are key enablers for organizations to identify quality knowledge which can be reused. It provides a better understanding of 
the quality of knowledge which could influence the improvements of individual job performance within the companies.

Problem Statement: Knowledge sharing and knowledge management are essential for organizations. According to Rai Nemani (2011), knowledge management is the recognition of the collection of the constant stream of information in an organizations day-to-day activity which can be utilized to help the organization to sustain its competitive advantage. Knowledge is the main asset for many companies because with good quality knowledge being shared across the organization, employees would be able to know what information they need, who to look for and what to do in their tasks. Knowledge centric organizations organize knowledge sharing sessions for their employees to share knowledge and use knowledge management systems to store and manage knowledge. However, employees might not be motivated to perform these knowledge sharing activities. They might be tied up to their work or afraid of what they shared might receive negative comments. On the other hand, some companies offered incentives or make it mandatory that employees share knowledge as part of the employees' key performance index (KPI). This method is not very effective as it forces employees to perform duties which are not to their liking. Research outcomes from McDermott and Dell (2001)'s work indicated that overcoming cultural barriers on knowledge sharing within large global organizations among their employees have always been difficult even though their systems, have features and functionalities are interesting and easy to use however these systems are rarely been used thoroughly. The website designer had interviewed users and they commented that the website was good and useful but the cultural resistance was the main issue.

Employees always commented that they don't have time to share knowledge. Besides this issue, employees would randomly share some news to fulfil their job requirement by sharing knowledge among other peers which may not be useful or relevant. Hence, the quality of knowledge shared might not be useful to other employee at all. In the end, the knowledge management system that is used to manage knowledge will contain lots of information that might not useful or it would be left unused. Therefore, the efforts for developing or adopting such a system would be meaningless. Moreover, another issue where knowledge management systems failed to improve organizational knowledge sharing would be knowledge hoarding. Knowledge hoarding happens when an employee withheld from sharing information among peers in order to ensure job security. As no employees would like to lose their jobs, they would protect definitely protect their job especially when the organization is downsizing. (Adobe Systems Incorporated, 2013). Furthermore, knowledge quality would drop as the knowledge they share might not be completed in order for them to secure their expertise. Employees would only share their expertise if they are guarantee of the job or they believe it to be 'safe'. In other words, organization culture plays an important role in ensuring employees is willing to accept the change from a knowledge hoarding culture to a collaborated knowledge sharing culture. Organizations are now looking into Web 2.0 technologies which were seen accelerating throughout the last few years for knowledge sharing. More and more companies have been using social networks within the organization. According to Knights (2007), many companies have already started using Web 2.0 technologies which has improved user productivity and increased customer feedback engagement through web-enabled front-end applications. By grabbing the data from social network sites and using tools such text miner, companies can analyse users' sentiments on that topic through sentiment network analysis. Sentiment network analysis is an analysis on public sentiments which are in the form of text.

According to Barber (2010), sentiments analysis is a type of natural language processing to identify the mood of public which involves the collection of opinions regarding a product made in social network sites such as tweets, comments or updates. Companies can derive knowledge from the sentiment network analysis. However, when using social network sites within a company, employees tend to mix up between personal and work related issues which could influence on the knowledge quality. Information shared might not be useful and over excessive. In Knights's (2007) article, according to Suw Charman, a social software consultant, social network sites might bring the opposite effect of sharing quality knowledge where it would end up having too much data. In short, knowledge sharing is essential and is beneficial to all knowledge workers in the companies. In this research, companies that adopt social network sites as the platform for knowledge management are studied. However, there might be some implications on the use of social network analysis for knowledge sharing when the presence of sentiment from posts entered by knowledge workers on the quality of knowledge and individual job performance. Therefore, this research will look into knowledge workers who use social network sites for knowledge sharing and 
analysing the content and sentiment to determine their influence to the quality of knowledge and their individual job performance.

Objectives: The objective of this research is to analyse Web 2.0 technologies and their influences on quality of knowledge and job performances among knowledge workers through the use of sentiment network analysis. The relationship between the participants of Web 2.0 technologies and their sentiments on social network sites provide an understanding of the presence of sentiments would facilitate better knowledge sharing, improvement on quality of knowledge as well as individual job performances within organizations. Based on the work conducted by Dasgupta \& Dasgupta (2009), the authors study the influences of social network using Web 2.0 on aspects such as the components, types and behavior of Web 2.0. One of the areas is the impact of social networks using Web 2.0 where the authors describe the implementation of social networking sites in a specific site called the Chicago. Every block has proven to help the organization in tracking criminals as well as protecting the public. A simple social networking site, which is easy to use, cost-effective can be used to fight crime. Hence, understanding the important factors where Web 2.0 technologies data can improve organization quality of knowledge is one of the main objectives of this research to help organizations to improve job performance.

Another objective of this research is to study and promote the knowledge sharing culture and analyze the behaviors of employees which could contribute to knowledge sharing. This also includes analyzing the different types of knowledge based on the content on the Web 2.0 technologies' data and how organizational employees are able to apply the knowledge to the work environment. According to works conducted by Marques et al (2008), the authors demonstrated that individual who share knowledge are more likely to improve performance within the workplace and also individuals who have better performance are more likely to share knowledge. This shows that knowledge sharing does influence job performance and the likelihood of having a knowledge sharing culture within the organization would increase. Thus, this research will study the employees' behaviour which would promote knowledge sharing where the quality of knowledge is going to help improve their work performance. Furthermore, through sentiment analysis, organizations will be able to analyse the positive and negative sentiments posted by the employees effectively and efficiently and generate the results in a simple visual presentation. The objective of this would be to use SAS Text Miner to analyse the data and produce this visual presentation or the statistics visually which would be easily viewable for the managers. This information could also be drilled down into specific details for further references. The pattern analysis that will be carried out on the system will analyse the posting patterns of the employees such as the users, the contents, users' behaviour and others. This way through the models, organizations would be able to have a high-level view of the information being shared through Web 2.0 technologies and the sentiments being discussed by participants. The information can be categorized structural data where companies can make improvements in knowledge quality and job performance.

\section{The Proposed Model}

The proposed research model for this study is shown in the following Figure 1. The proposed model investigates the influence of sentiment toward quality of knowledge and quality of knowledge toward individual job performance.

\section{Figure 1: Proposed Research Model}

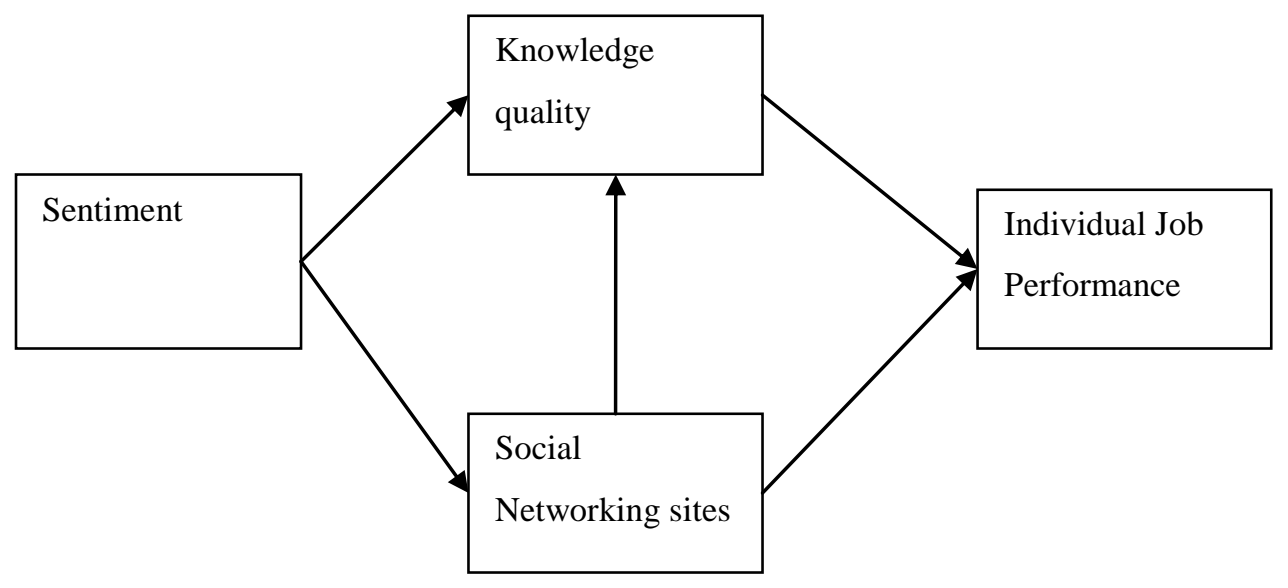


The proposed model uses Sentiment Network Analysis as the basic theory to investigate the presence of sentiment in a social network and its effect toward the knowledge quality as knowledge workers communicate and collaborate to each other. Such effect may positively influence the individual job performance hence may either improve or not improve the company's productivity. The proposed model also looks at the social networking sites, in this case, micro blogging systems, and its effect toward individual job performance.

\section{Methodology}

This research carries out a detailed analysis on Web 2.0 technologies and the social media data is analyzed using text mining techniques and sentiment analysis to understand the sentiments of the posts from participants on the social network sites. One of the techniques to analyze sentiments is to use sentiment network analysis which will reveal the influence of sentiment among participants toward the knowledge shared and created among them. Firstly, this research studies and reviews on Web 2.0 technologies to gain an understanding on the background of Web 2.0 technologies as well as its adoption by company which made use of it to manage knowledge. Based on this understanding, a specific company that uses social network sites as their platform for knowledge sharing is selected for this research. Data collection method such as focus group studies, interviews and questionnaires will be carried out to gather accurate data on the employee's view and use on the Web 2.0 technologies within their company. A sample of 10 to 15 employees across the organization is selected to conduct an interview to understand their nature of business related to knowledge sharing and the use of the social network sites. Employees from different level of management would have different opinions or knowledge to share. The usage of the social network sites too would differ between employees as some might departments might share more information compared to others. In addition, questionnaires will be handed out to a larger group to other employees consisting around 20 to 30 people to obtain more information on employees perception towards the knowledge quality shared on the social network sites which improves their job performance.

Moreover, in this research SAS Enterprise Miner tool which provides text mining capabilities is used to analyze the topics converse through the social network sites. Pattern analysis will be carried out on the employees' entries in the system using the SAS software to identify different patterns that could be beneficial to their work such as job performance improvements. Through the use of SAS Text Miner, I analyze the data which comes from the social network sites to determine the positive and negative sentiments. Sentiment analysis requires an adequate understanding of the particular person feelings, emotions and behavior. Therefore, to derive high quality information from text, the outcomes will be used to determine whether the entries in social network sites influences the employees work performance. With the topic analysis and also conducting a concept linking through the SAS software, this research could draw out a more accurate and interesting kind of finding and patterns from the entries of the organizations social network analysis. Throughout the analysis of the social network sites, SAS Text Miner will be able to identify the possible patterns. Based on the results, quality of knowledge improvements that could improve and increase job performance for knowledge workers could be identify.

\section{Conclusion}

Based on this research, the company can derive from the result whether employees' sentiments through the social network analysis influences knowledge quality and their job performance. By sharing knowledge and communicating through the social network sites, employees' entries are gathered to understand the quality of knowledge shared. The sentiments mined through text miner and sentiment analyser are able to show the impact of quality of knowledge shared on their individual job performance.

\section{References}

Adobe Systems Incorporated. (2013). Why Your Organization Should Support a Knowledge Sharing Culture [online] http://blogs.adobe.com/captivate/2013/01/why-your-organization-shouldsupport-a-knowledge-sharing-culture.html [Accessed on 18 th September 2013].

Barber, I. (2010). Opinion mining - sentiment mining [online] http://searchbusinessanalytics.techtarget.com/definition/opinion-mining-sentiment-mining [Accessed on $20^{\text {th }}$ September 2013]. 
Costa, P. R. S., Souza, F. F., Times, V. C. \& Benevenuto, F. (2012). Towards Integrating Online Social Networks and Business Intelligence, In Proceedings of the IADIS International Conference on Web Based Communities and Social Media (WBC'12). Lisbon, Portugal, July 2012.

Dasgupta, D. \& Dasgupta, R. (2009). Social Networks using Web 2.0 [online] http://www.ibm.com/developerworks/webservices/library/ws-socialcollab/ [Accessed on 23 rd September 2013].

Grabowicz, P. (2013). The transition to digital journalism [online] http://multimedia.journalism.berkeley.edu/tutorials/digital-transform/web-20/ [Accessed on 29th August 2013].

Grit, J. W. (2009). Stumble upon hidden Knowledge: Micro blogging in Organizations [online] http://essay.utwente.nl/59557/1/scriptie_J_Grit.pdf [Accessed on 4 ${ }^{\text {th }}$ August 2013]

Gunther, O., Krasnova, H., Riehle, D. \& Schoendienst, V. (2009). Modeling Micro blogging Adoption in the Enterprise [online] http://www.icsi.berkeley.edu/pubs/other/modelingmicroblogging09.pdf [Accessed on $10^{\text {th }}$ August 2013].

Jacques B., Chui, M. \& Miller, A. (2009). How companies are benefiting from Web 2.0: McKinsey Global Survey results [online] http://www.mckinsey.com/insights/business_technology/ how_companies_are_benefiting_from_web_20_mckinsey_global_survey_results [Accessed on $10^{\text {th }}$ August 2013].

$\begin{array}{lllllll}\text { Knights, } & \text { M. } & \text { (2007). Using } & \text { Web } & \text { for } & \text { business }\end{array}$ http://www.computerweekly.com/news/2240082941/Using-Web-20-for-business [Accessed on $16^{\text {th }}$ August 2013].

Liu, B. (2010). Sentiment analysis and Subjectivity [online] http://people.sabanciuniv.edu/berrin/proj102/1-BLiu

Sentiment\%20Analysis\%20and\%20Subjectivity-NLPHandbook-2010.pdf $\quad\left[\right.$ Accessed $\quad$ on $18^{\text {th }}$ September 2013].

Marques, D. V., Cardoso, L. \& Zappala, S. (2008). Knowledge sharing networks and performance [online] http://www.scielo.oces.mctes.pt/pdf/cog/v14n2/v14n2a04.pdf [Accessed on 23 $3^{\text {rd }}$ September 2013].

McDermott, R. \& O’Dell, C. (2001). Overcoming cultural barriers to sharing knowledge [online] http://practicestories.ca/sites/default/files/Document\%20Library/overcoming_cultural_barrier s_to_sharing_knowledge.pdf [Accessed on 26 $6^{\text {th }}$ September 2013].

Nemani, R. (2011). Knowledge Management and Knowledge Sharing [online] http://www.b-eyenetwork.com/view/15293 [Accessed on 29th August 2013].

Prabowo, R. \& Thelwall, M. (2003). Sentiment Analysis: A Combined Approach [online] http://www.cyberemotions.eu/rudy-sentiment-preprint.pdf [Accessed on $20^{\text {th }}$ September 2013]. 